Relations industrielles

Industrial Relations

\title{
Lallement, Michel, sous la direction de, Travail et emploi: le temps des métamorphoses
}

\section{Denis Harrisson}

Volume 50, numéro 2, 1995

URI : https://id.erudit.org/iderudit/051026ar

DOI : https://doi.org/10.7202/051026ar

Aller au sommaire du numéro

Éditeur(s)

Département des relations industrielles de l'Université Laval

ISSN

0034-379X (imprimé)

1703-8138 (numérique)

Découvrir la revue

Citer ce compte rendu

Harrisson, D. (1995). Compte rendu de [Lallement, Michel, sous la direction de, Travail et emploi: le temps des métamorphoses]. Relations industrielles /

Industrial Relations, 50(2), 458-461. https://doi.org/10.7202/051026ar

Tous droits réservés @ C Département des relations industrielles de l'Université Laval, 1995
Ce document est protégé par la loi sur le droit d'auteur. L’utilisation des services d'Érudit (y compris la reproduction) est assujettie à sa politique d'utilisation que vous pouvez consulter en ligne.

https://apropos.erudit.org/fr/usagers/politique-dutilisation/ 
reason why action (internally) has been initiated is because of a change in some external variable. It is the role of scholars to theorize and 'develop' such linkages, to provide an understanding or explanation of real world phenomena.

Second, as Blackmur's title suggests, one of the contributions of his book is to consider the 'consequences' of the two disputes. However he finds himself trapped by his methodology. Because he hasn't actually examined the historical record (and in considering 'consequences' where does one stop?), other than immediately after the cessation of the disputes, he can only provide a 'preliminary' explanation, offer suggestions (is this akin to theorizing?), raise questions and state that the book has been only able to scratch the surface of a potentially highly fruitful field of research' (p. 206). Does this mean that after ten or fifteen years of enquiry, he feels compelled to pass the baton on to someone else?
Industrial relations scholarship has often been criticized for its lack of a theoretical orientation - or what Blackmur might refer to as 'impotent empiricism' (p. 212). It will be asserted here that a necessary prerequisite of a discipline, and of scholars who inhabit its space, is to indulge and experiment in theoretical discourse, to develop and test theories. In a sense all social phenomena is of the order of 'significant unforeseen consequences'. One isn't an historian to celebrate a methodology. The essential epistomological function of historical scholarship is that it provides empirical data upon which theories can be tested and developed, thereby providing us with an understanding of the real world of 'significant unforeseen consequences'. To return to the question of how powerful a tool is the mind - if you don't try to use it, you will never find out!

BRAHAM DABSCHECK

The University of New South Wales

\section{Travail et emploi: le temps des métamorphoses}

sous la direction de Michel LALLEMAND, Paris, L'Harmattan, Collection "Logiques sociales ", 1994, 283 p., ISBN 2-7384-2559-3.

Ce volume rassemble les contributions de 11 auteurs qui analysent les transformations en cours dans l'emploi et le travail. C'est un ouvrage ambitieux car le défi que les auteurs se sont imposés est considérable. Il s'agit de dresser un bilan des changements de l'emploi et du travail tant au plan de la dynamique des organisations que des mutations dans le marché du travail, puis de présenter les nouvelles voies théoriques qui s'ouvrent afin d'analyser les configurations sociales et économiques récentes dans deux disciplines, l'économie et la sociologie. Avec un tel projet, peut être un peu trop ample pour un seul volume, le lecteur comprendra que chaque chapitre couvre de façon succincte les différents aspects abordés selon l'expertise de l'auteur. Les sujets traités sont universels mais la France sert d'exemple pour la dimension empirique. Le lecteur trouvera dans ce volume une analyse des principales conséquences de la mise en place d'un nouvel ordre économique pour l'emploi et le travail.

La première partie est divisée en six chapitres dans lesquels différents auteurs traitent du chômage comme d'un phénomène permanent (chap. I), des nouvelles formes d'organisation du travail et de l'entreprise (chap. II), de la technologie dans ses rapports à l'emploi et 
aux qualifications (chap. III), des relations professionnelles (chap. IV), des transformations du secteur tertiaire dans ses rapports à l'emploi (chap. V) et de la politique salariale de l'État (chap. VI).

Un constat traverse l'analyse de ces différents éléments : le chômage est un fait qui perdure, ce qui pose à la fois un défi pragmatique pour tenter de réduire ce phénomène tragique et un défi théorique afin de réviser les catégories analytiques des effets du sous-emploi. La période actuelle se caractérise par une opposition entre un chômage répétitif marqué par des passages fréquents d'une situation d'emploi au non emploi et un chômage d'exclusion d'une durée très longue. Les inégalités et les disparités spatiales, de sexe et d'âge montrent l'hétérogénéité du chômage et rendent complexes les politiques de résorption. Ces mutations du chômage sont le résultat partiel des transformations des formes de l'organisation du travail et de l'entreprise, du processus de production et également des normes de consommation. Ces tendances dans l'entreprise n'ont pas encore atteint leur forme définitive mais l'ampleur macro-économique et sociale du phénomène montre bien l'importance d'en prendre la mesure.

L'argument de la polarisation des qualifications suite aux changements technologiques a produit des thèses diversifiées. L'effet sociétal dans la définition de la qualification et de ses rapports aux systèmes éducatif, organisationnel et industriel renouvelle la perspective théorique traitant des modes de régulation du système productif plutôt que de progrès technique. Le déterminisme technologique "doux " influe non pas sur les qualifications mais sur une notion à développer davantage, les " compétences".

Les relations professionnelles et la gestion de l'emploi interviennent également dans le débat. Les nouveaux accords négociés tendent à renforcer l'efficacité et la compétitivité de l'entreprise par un réarrangement approprié des conditions de travail des salariés. Ces accords président à l'émergence d'une nouvelle problématique où la négociation collective servirait davantage à l'amélioration de la situation de l'entreprise qu'à celle des travailleurs. La flexibilité au nom de laquelle les mutations industrielles se réalisent conduit à une plus grande influence de la situation de l'entreprise et à une plus forte individualisation des salaires qui s'inscrit dans de nouvelles pratiques de gestion de la main-d'œuvre des entreprises.

Alors que dans plusieurs secteurs économiques on assiste à des pertes massives d'emploi, le secteur tertiaire en crée. Mais il faut s'interroger sur la capacité des services à impulser une dynamique de croissance. Pour les "traditionalistes", les emplois du secteur tertiaire font partie d'un processus déséquilibrant alors que pour les "modernistes ", la création d'emploi dans les services caractérise un moment de retour vers une croissance "vertueuse" qui impose une nouvelle analyse. En effet, pour être au centre d'une croissance équilibrée, les emplois tertiaires doivent refléter des choix sociétaux qui permettent la recomposition de nouveaux rapports entre les secteurs industriel et tertiaire. Sans nouvelle régulation sociétale, la croissance qu'impose le secteur tertiaire pourrait bien n'être qu'artificielle.

Voilà brièvement exposés les arguments présentés dans cette première partie qui trace le bilan des mutations de l'emploi et de leurs principaux déterminants. Comme il est fréquent dans ce genre d'ouvrage faisant appel à différents auteurs, les chapitres, hélas, ne sont pas de qualité égale. Certains auteurs structurent mieux leur argumentation que d'autres et ils savent aller à l'essentiel sans sacrifier ni la clarté, ni l'intensité de leur exposé. Dans certains chapitres, les auteurs se contentent de tracer un bilan sans rien ajouter de nouveau à ce que le lecteur avisé sait déjà. Le tour d'horizon des thèses et débats est 
rapide, l'analyse est trop souvent superficielle et le lecteur est laissé à lui-même pour porter un jugement sur le bilan. C'est le cas des chapitres sur l'organisation du travail qui consacre trop d'espace au bilan du taylorisme et celui sur les nouvelles technologies qui présente avec trop d'insistance les débats d'hier et sacrifie la présentation de la discussion actuelle sur les "compétences". Dans d'autres chapitres, les auteurs se sont efforcés de tracer des avenues nouvelles et d'aller au-delà des interprétations courantes. Le chapitre sur le chômage, celui sur l'emploi tertiaire et celui sur les relations professionnelles sont des moments forts de cette première partie de l'ouvrage. Par ailleurs, nous déplorons l'absence de synthèse véritable des apports de la sociologie du travail et de l'économie du travail dans ce bilan. En effet, la formation d'économiste ou de sociologue des auteurs teinte fortement la lecture des mutations du travail et de l'emploi et l'on passe d'une interprétation d'économiste ou de sociologue sans véritable intégration.

Cinq chapitres couvrent la deuxième partie consacrée aux nouveaux paradigmes en sociologie et en économie du travail : deux chapitres (Chap. VII et IX) présentent les apports de l'économie du travail, deux autres chapitres (Chap. VIII et $\mathrm{X}$ ) traitent des contributions de la sociologie et un dernier chapitre (Chap. $\mathrm{XI}$ ) tente une synthèse interdisciplinaire. La trame principale est bien suivie dans cette partie théorique du volume. Il s'agit de tenir compte de l'existence d'un niveau de chômage élevé pour renouveler l'analyse du marché du travail.

Suite à un rapide tour d'horizon des grands paradigmes classiques de l'économie du travail et des théories courantes, l'approche de la segmentation du marché du travail est présentée pour sa contribution à l'émergence de nouvelles théories de la relation d'emploi qui seraient soucieuses de l'autonomie relative des marchés du travail et de leur impact sur les déséquilibres macro-économiques.
Parmi les nouveaux développements théoriques, la conceptualisation qui repose sur la notion de convention propose l'abandon de la thèse de l'efficacité du marché autorégulateur au profit de la réhabilitation des règles et des organisations au sein de la théorie économique. Les rapports entre les agents économiques nécessitent une stabilité que l'on retrouve dans les contrats qui sont des formes objectivées d'interactions sociales. Ainsi dans la relation d'emploi, le principe d'organisation s'avère plus efficace que le principe de marché. Ce chapitre (IX) est l'un des meilleurs de ce volume. Loin d'être complaisant envers la nouveauté, les auteurs présentent également les critiques à cette approche française controversée, notamment sur la dimension du conflit présentée par les théoriciens des conventions comme une tension alors qu'il semble etre au centre de l'échange et du principe économique marchand.

Faisant écho à la théorie économique des conventions, les théories de l'acteur sont réhabilitées en sociologie du travail. Inspiré par l'interactionnisme symbolique, les phénomènes sociaux sont analysés comme le résultat d'un ensemble d'actions individuelles ou institutionnelles. Malheureusement, ce chapitre (X) tombe à plat. En tentant de présenter quatre écoles de pensée, l'auteur dilue l'approche et ne réussit pas à convaincre le lecteur de l'importance des théories de l'acteur. Les dimensions historiques occupent un espace démesuré et les positions actuelles de ces théories sont rapidement esquissées vers la fin par l'illustration de quelques exemples trop peu convaincants. Le travail est présenté comme un construit social qui s'inscrit dans des rapports économiques et constitutifs des sociétés contemporaines. Par ailleurs, les contributions à l'étude de l'emploi sont rares et incomplètes. C'est pourquoi une nouvelle sociologie de l'emploi, située à l'intersection de l'économie et de la sociologie, pourrait se consacrer à l'analyse de la 
construction sociale des marchés du travail et des rapports sociaux de l'emploi, à partir desquels s'organisent les clivages sociaux dans l'entreprise.

Les questions soulevées suite aux nombreux changements dans le travail et l'emploi méritaient certainement qu'un volume y soit consacré. La démarche choisie par les auteurs consiste en un traitement analytique à l'aide de plans dualistes : tracer un bilan factuel et intellectuel, traiter des métamorphoses du travail et de l'emploi, s'appuyer sur les travaux en sociologie et en économie du travail. Avec un tel projet, les auteurs sont forcément obligés de tracer à grand trait leur argumentation. Les parties sur les nouvelles avenues de recherche et sur les nouveaux développements théoriques souffrent le plus de ces ellipses intellectuelles. Néanmoins il s'agit d'un ouvrage important pour le traitement original de l'ensemble de ces questions autour des notions de convention, d'interaction et de négociation. La lecture est agréable, certains chapitres sont fort relevés et une bibliographie substantielle supplée aux lacunes de la démonstration. Quiconque s'intéresse à ces questions devrait lire ce volume.

DENIS HARRISSON

Université du Québec à Hull

\section{Home to Work: Motherhood and Politics of Industrial Homework in the United States}

by Eileen BORIS, Cambridge, UK, Cambridge University Press, 1994, 383 p., ISBN 0-521-44370-9.

This book is an historical overview of waged (industrial) labour in the U.S An interesting aspect of the book is that it connects homework of 1880 s to the present day homework of white-collar female employment. It shows the similarities of working at home as waged labour then and now, and weaves this within a feminist framework.

The book presents the view that workplaces have been organized without considering the parental identity of workers. The workplace assumes that most workers are men with a supporting woman at home. In her book, Boris combines feminist theory and historical research to address the "double day" of working women, how "home" and "work" interconnect despite their ideological separation. The book also shows how 1980s deregulation of homework in the U.S. brought past and present together.

The author's purpose in this book is not to give a precise economic or social history. Her purpose is to discuss the social construction of concepts of wom- anhood and manhood, visions of proper homelife and childhood, and how the state policy separated home from work. The book is illustrating the historical construction of gender and the gendering by giving examples of the state and its actions through legislation and regulation.

Boris shows in her book that we have "the history of labor law and the history of protective labor legislation for women and children, but the gendered assumptions of the former remain unrevealed in major studies" (p. 7). The book fulfills its purpose by examining how, in the U.S. context, policies such as women's labour laws and maternal health care had gendered assumptions; and how middle- and upper-class white women's culture, through their organizations, influenced state politics creating the belief in female difference. Starting in late nineteenth century the concept of difference assigned women superior moral and personal qualities, such as nurturing, cooperativeness and selflessness; giving women the major responsibility of 